\title{
Revisiting Rotor Rigid Body Modes: Parametric Study of Stability
}

\author{
NATHAN B. LITTRELL, AGNES MUSZYNSKA* and PAUL GOLDMAN \\ Bently Rotor Dynamics Research Corporation, P.O. Box 2529, Minden, NV 89423-2529, USA
}

(Received 16 April 1998; In final form 20 June 1998)

\begin{abstract}
This paper presents an analytical model of a rigid rotor supported in two fluid film bearings with an emphasis on predicting the instability threshold speed. The factors contributing to the stability of the rotor are discussed and presented graphically using root locus plots. The parametric study of the stability starts from the discussion of the rotor/bearing system with "mirror symmetry". Three basic cases are considered:
\end{abstract}

(i) Rotor with relatively small gyroscopic effect (small polar moment of inertia) and relatively high transverse moment of inertia. It is found that the pivotal mode instability exists, but the lateral mode controls stability.

(ii) Highly gyroscopic rotor (relatively large polar moment of inertia) with also relatively low transverse moment of inertia. It is found that the pivotal mode is infinitely stable and the lateral mode controls stability.

(iii) Highly gyroscopic rotor with relatively high transverse moment of inertia. It is found that the pivotal mode exists and controls stability. The lateral mode always exists.

Both asymmetry in rotor geometry (location of center of mass with respect to the bearings) and fluid bearing parameters (stiffness, damping) are considered. It is shown that, for a given bearing asymmetry parameter, the maximum stability is achieved when the geometric asymmetry parameter is of equal value. The recommendations on the optimal design from the stability standpoint are given.

Keywords: Gyroscopic, Fluid bearing, Optimal design, Stability, Root locus

\section{INTRODUCTION}

The gyroscopic effect, as related to rotordynamics, has been researched in many papers, starting from pioneering works by Yamamoto
(1954), Dimentberg (1961) and Crandall (1982, 1961) considering various ways of describing the rotor lateral and angular motion. A description of gyroscopic effects together with more complete lists of references can be found in books by

*Corresponding author. Tel.: 702 782-3611. Fax: 702 782-9236. E-mail: agnes@brdrc.com. 
Ehrich (1992) and Vance (1988). An experimental work dealing with parameter identification for the rotor system with large gyroscopic influence is reported by Bently et al. (1986). The interaction between the stabilizing effect of gyroscopics and destabilizing effect of fluid-induced tangential forces has been investigated by Muijderman (1986).

An earlier paper by Hatch and Bently (1995) presented a model and stability criteria for a rigid rotor with significant gyroscopic effects supported in one fluid film bearing and one roller element bearing. The latter was assumed to have infinite lateral stiffness. The significant finding of this paper was that it is possible to make a rotor pivotal mode absolutely resistant to fluid induced instabilities by using the gyroscopic effect to counteract the forces driving the fluid instability. Experimentation verified the conclusions drawn in this paper, leading to the feasibility of employing the stability criteria on real machines to correct fluid instability problems.

One of the questions that came up was, "what if a machine has two fluid film bearings?" By adding another fluid film bearing, it is expected that there will be a translational mode as well as a pivotal mode. The second fluid film bearing introduces a new, strictly lateral, degree of freedom, and another mode. Investigating the stability criteria for this more complex model was the motivation for this study.

\section{ROTOR MODEL}

A diagram of the rotor modeled is shown in Fig. 1. The model consists of a rotor supported in two fluid lubricated bearings with fluid film radial stiffness $K_{\mathrm{b}}$, and rotating damping $D_{\mathrm{b}}$, subscripted with a 1 or 2 to indicate which bearing is referred to. The rotor itself has parameters of mass $M$, polar mass moment of inertia $I_{\mathrm{p}}$, and transverse mass moments of inertia $I_{x}, I_{y}$.

The coordinate system for describing the rotor motion is shown in Figs. 2 and 3. It is a combination of a Cartesian and a spherical system. The basis of the absolute stationary coordinate system $x y z$ has

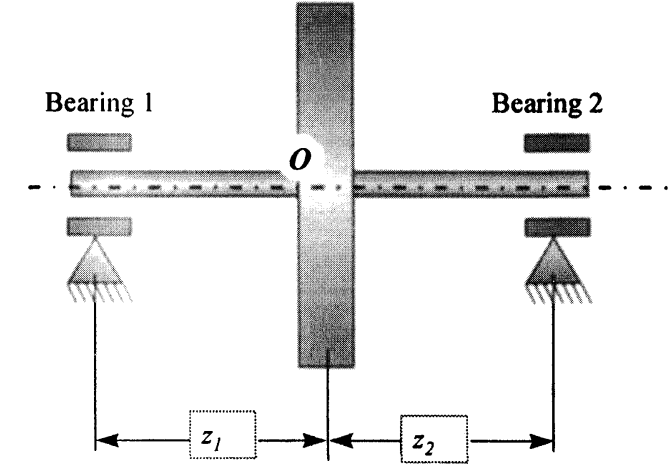

FIGURE 1 Diagram of rotor system used in model.

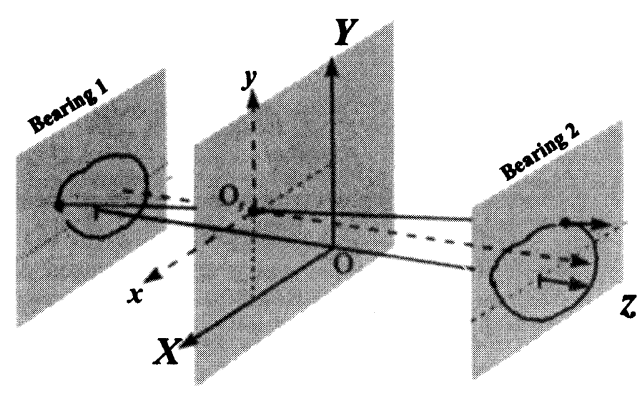

FIGURE 2 Coordinate systems for the rigid rotor.

its origin at the point $\mathrm{O}$ which is coincident with the rotor mass center, $O_{\mathrm{r}}$, when the rotor is centered in the bearing clearances. The translational motion of the rotor is assumed planar, and is described by lateral displacements $x$ and $y$. Additionally, a spherical coordinate system is introduced with origin at the rotor mass center $O_{\mathrm{r}}$, and $\chi$ and $\psi$ as angles of yaw and pitch respectively (Fig. 3). Note that for the rotor as shown in Fig. 1, distance $z_{1}$ is negative. Of course, generally a rotor may have its mass center outside of the bearing span in which case $z_{1}$ and $z_{2}$ are both of the same sign. Such rotors are usually called "overhung" rotors.

Any lateral displacement of the rotor relative to its center of mass, $O_{\mathrm{r}}$, can be described by the two angles plus the distance $z$ from $O_{\mathrm{r}}$. The combination of the $(x, y)$ and $(\chi, \psi)$ coordinate systems provide the independent coordinates used for a Lagrangian 


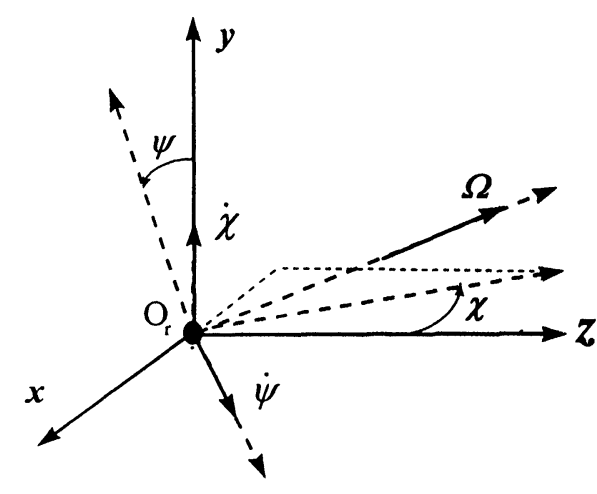

FIGURE 3 Angles $\chi, \psi$ of yaw and pitch.

derivation of the equations of motion (not presented in this paper).

\section{Assumptions}

- Bearing fluid film stiffness and damping properties are considered laterally isotropic. This is true for the case of lightly loaded bearings/ seals.

- The rotor is assumed rigid.

- It is assumed that the angular displacement of the rotor is small, consequently the distance from the origin along the axis is maintained as simply $z$. Additionally, gyroscopic effects of second order or higher are neglected.

- There is no axial motion of the rotor.

- The fluid film damping in the bearings is directly proportional to the fluid film stiffness.

- The fluid circumferential average velocity ratio, $\lambda$ (Muszynska, 1988), is considered the same in both bearings.

- The rotative speed $\Omega$, of the rotor is assumed constant. There is no torsional vibration. All calculations consider variation of rotor speed discretely.

\section{Equations of Motion}

The four degrees of freedom $x, y, \chi$ and $\psi$ can be reduced to two by introduction of the complex coordinates $r=x+\mathrm{j} y$, and similarly, $\Psi=\chi+\mathrm{j} \psi$. Using these identities, the equations for the free response of the system are as follows:

$$
\begin{aligned}
& M \ddot{r}+\left(D_{\mathrm{b} 1}+D_{\mathrm{b} 2}\right) \dot{r}+\left[\left(K_{\mathrm{b} 1}+K_{\mathrm{b} 2}\right)\right. \\
& \left.\quad-\mathrm{j} \lambda \Omega\left(D_{\mathrm{b} 1}+D_{\mathrm{b} 2}\right)\right] r \cdots+\left(D_{\mathrm{b} 2} z_{2}+D_{\mathrm{b} 1} z_{1}\right) \dot{\Psi} \\
& \quad+\left[K_{\mathrm{b} 2} z_{2}+K_{\mathrm{b} 1} z_{1}-j\left(D_{\mathrm{b} 2} z_{2}+D_{\mathrm{b} 1} z_{1}\right) \lambda \Omega\right] \Psi=0,
\end{aligned}
$$

$$
\begin{aligned}
I_{\mathrm{t}} \ddot{\Psi}+ & {\left[\left(D_{\mathrm{b} 1} z_{1}^{2}+D_{\mathrm{b} 2} z_{2}^{2}\right)-\mathrm{j} I_{\mathrm{p}} \Omega\right] \dot{\Psi}+\left[\left(K_{\mathrm{b} 1} z_{1}^{2}+K_{\mathrm{b} 2} z_{2}^{2}\right)\right.} \\
& \left.-\mathrm{j} \lambda \Omega\left(D_{\mathrm{b} 1} z_{1}^{2}+D_{\mathrm{b} 2} z_{2}^{2}\right)\right] \Psi+\left(D_{\mathrm{b} 2} z_{2}+D_{\mathrm{b} 1} z_{1}\right) \dot{r} \\
& +\left(K_{\mathrm{b} 2} z_{2}+K_{\mathrm{b} 1} z_{1}-\mathrm{j} \lambda \Omega\left(D_{\mathrm{b} 2} z_{2}+D_{\mathrm{b} 1} z_{1}\right)\right) r=0,
\end{aligned}
$$

where $\lambda$ is the fluid circumferential average velocity ratio as shown by Muszynska (1988). In order to reduce the complexity of the equation set and possibly gain some insight into the physical behavior of the rotor system, nondimensional forms of Eqs. (1) and (2) are generated using the relations given in Table $\mathrm{I}$ :

$$
\begin{gathered}
h^{\prime \prime}+2 \zeta_{\mathrm{t}} h^{\prime}+\left(1-2 \mathrm{j} \zeta_{\mathrm{t}} \lambda \omega\right) h \\
+b_{\mathrm{t} \theta}\left(\theta^{\prime}-\mathrm{j} \lambda \omega \theta\right)+a_{\mathrm{t} \theta} \theta=0, \\
\Psi^{\prime \prime}-\mathrm{j} \frac{\omega}{\kappa} \Psi^{\prime}+2 \eta \zeta_{\theta}\left(\Psi^{\prime}-\mathrm{j} \lambda \omega \Psi\right)+\eta^{2} \Psi \\
+\frac{b_{\mathrm{t} \theta}}{\kappa}\left(h^{\prime}-\mathrm{j} \lambda \omega h\right)+\frac{a_{\mathrm{t} \theta}}{\kappa} h=0 .
\end{gathered}
$$

\section{STABILITY OF THE MIRROR SYMMETRIC SYSTEM-UNCOUPLED MODES}

The rotor system (Fig. 1) is considered "mirror symmetric" if the distance from the center of mass to either bearing is identical $\left(\left|z_{1}\right|=\left|z_{2}\right|\right)$ and the bearing characteristics are the same $\left(D_{\mathrm{b} 1}=\right.$ $\left.D_{\mathrm{b} 2}, K_{\mathrm{b} 1}=K_{\mathrm{b} 2}\right)$. In that case the cross-coupling factors, $a_{\mathrm{t} \theta}$ and $b_{\mathrm{t} \theta}$, vanish and translational and pivotal modes, Eqs. (3) and (4) become uncoupled.

(1) For translational mode:

$$
h^{\prime \prime}+2 \zeta_{\mathrm{t}} h^{\prime}+\left(1-2 \mathrm{j} \zeta_{\mathrm{t}} \lambda \omega\right) h=0 .
$$


TABLE I Nomenclature of factors used to nondimensionalize equations of motion

Radius of gyration, $\rho$
Nondimensional displacement, $h$
Ratio of transverse to polar moment of inertia, $\kappa$
Lateral natural frequency, $\nu_{t}$
Angular natural frequency, $\nu_{\theta}$
Lateral damping factor, $\zeta t$
Angular damping factor, $\zeta_{\theta}$
Natural frequency ratio, $\eta$
Stiffness cross coupling factor, $a_{t \theta}$
Nondimensional rotor speed, $\omega$
Nondimensional time, $\tau$
Damping cross coupling factor, $b_{t \theta}$

$$
\begin{gathered}
\rho=\sqrt{I_{p} / M} \\
h=r / \rho \\
\kappa=I_{t} / I_{p} \\
\nu_{t}=\sqrt{K_{b 1}+K_{b 2} / M} \\
\nu_{\theta}=\sqrt{K_{b 1} z_{1}^{2}+K_{b 2} z_{2}^{2} / I_{t}} \\
\zeta_{t}=D_{b 1}+D_{b 2} / 2 \sqrt{\left(K_{b 1}+K_{b 2}\right) M} \\
\zeta_{\theta}=D_{b 1} z_{1}^{2}+D_{b 2} z_{2}^{2} / 2 \sqrt{\left(K_{b 1} z_{1}^{2}+K_{b 2} z_{2}^{2}\right) I_{t}} \\
\eta=\nu_{\theta} / \nu_{t} \\
a_{t \theta}=K_{b 2} z_{2}+K_{b 1} z_{1} / \rho\left(K_{b 1}+K_{b 2}\right) \\
\omega=\Omega / \nu_{t} \\
\tau=\nu_{t} \mathrm{~d} / \mathrm{d} \tau={ }^{\prime} \\
b_{t \theta}=D_{b 2} z_{2}+D_{b 1} z_{1} / \rho \sqrt{\left(K_{b 1}+K_{b 2}\right) M}
\end{gathered}
$$

(2) For pivotal mode:

$$
\theta^{\prime \prime}-\mathrm{j} \frac{\omega}{\kappa} \theta^{\prime}+2 \eta \zeta_{\theta}\left(\theta^{\prime}-\mathrm{j} \lambda \omega \theta\right)+\eta^{2} \theta=0
$$

The instability threshold speed for the translational mode is as follows:

$$
\Omega_{\mathrm{th}}^{(\mathrm{t})}=\frac{\nu_{\mathrm{t}}}{\lambda}=\frac{1}{\lambda} \sqrt{\frac{K_{\mathrm{b} 1}+K_{\mathrm{b} 2}}{M}} .
$$

From Eq. (7) it can be seen that the stability of the translational mode can be maximized three ways: Increasing direct stiffness values, decreasing the modal mass, $M$, or decreasing the bearing fluid circumferential average velocity ratio, $\lambda$. All will raise the speed at which the translational mode becomes unstable. There is no unconditional stability for the translational mode. The instability threshold speed for the pivotal mode is as follows:

$$
\Omega_{\mathrm{th}}^{(\theta)}=\frac{\nu_{\theta}}{\lambda \sqrt{\sigma}}=\frac{1}{\lambda} \sqrt{\frac{K_{\mathrm{b} 1} z_{1}^{2}+K_{\mathrm{b} 2} z_{2}^{2}}{I_{\mathrm{t}}-\left(I_{\mathrm{p}} / \lambda\right)}} .
$$

The interesting result from Eq. (8) is that an unconditional stability criterion can be derived using finite and achievable parameters as shown by Hatch and Bently (1995). By setting the term in the denominator under the radical less than or equal to zero, the following unconditional stability criterion is reached:

$$
\frac{I_{\mathrm{t}}}{I_{\mathrm{p}}} \leq \lambda
$$

Figure 4 shows a root locus plot of the rotor system with the criterion of Eq. (9) satisfied. By examining the roots of each of the decoupled equations (5) and (6), it can be seen that the lateral mode is the only source of the instability. A quick check of this is provided by observing that the roots generated by the gyroscopic term have a branch that is asymptotically approaching the zero real axis.

In Fig. 5 it can be seen that the lateral roots are unchanged, but now the gyroscopic root crosses the zero axis at lower rotative speed than the lateral root, thus controlling stability. This is due to the criterion set in (9) not being met. It should be noted that the stability of the gyroscopic mode does not necessarily have to be absolute, just better than the lateral mode, as the lowest threshold dominates. 


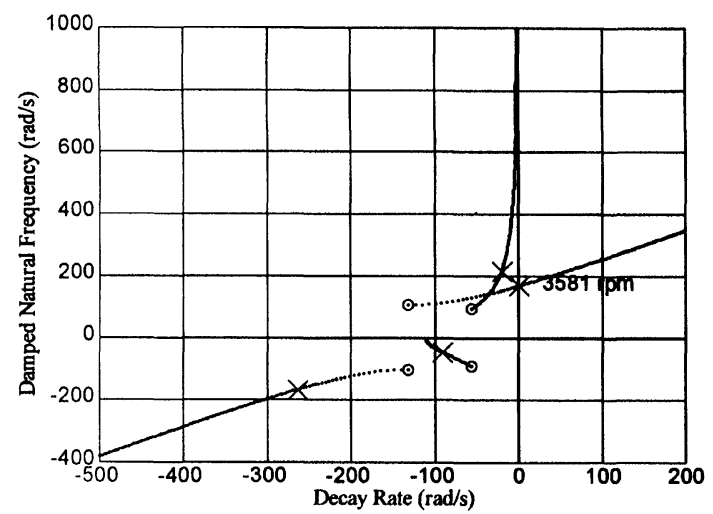

\begin{tabular}{|l} 
SYSTEM PARAMETERS \\
$M=2 \quad \mathrm{~kg}$ \\
$K_{b 1}=28 \mathrm{e} 3 \mathrm{~N} / \mathrm{m}$ \\
$K_{b 2}=28 \mathrm{e} 3 \mathrm{~N} / \mathrm{m}$ \\
$D_{b 1}=262.5 \mathrm{~N} \mathrm{~s} / \mathrm{m}$ \\
$D_{b 2}=262.5 \mathrm{~N} \mathrm{~s} / \mathrm{m}$ \\
$z_{1}=-0.102 \mathrm{~m}$ \\
$z_{2}=0.102 \mathrm{~m}$ \\
$\lambda=0.45$ \\
$I_{p}=0.022 \mathrm{~kg} \mathrm{~m}^{2}$ \\
$I_{t}=I_{p} / \lambda \mathrm{kg} \mathrm{m}^{2}$
\end{tabular}

FIGURE 4 Root locus plot of "mirror symmetric" rotor with ratio $\kappa=1 / \lambda$. Plot was generated using the parameters given above and varying rotative speed, $\Omega$. The O's indicate the beginning of the locus at $\Omega=0$. The $\times$ 's indicate the position of the roots at the point where the system becomes unstable at $\Omega=3581 \mathrm{rpm}$.

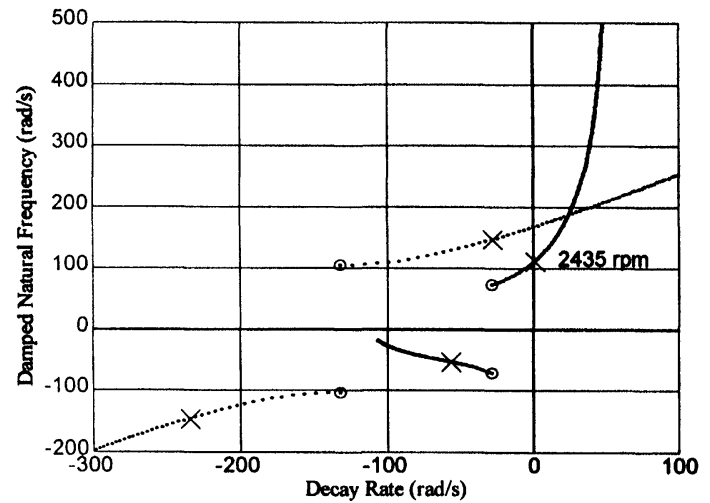

FIGURE 5 Root locus plot of symmetric rotor with system parameters as in Figure 4, except for the parameter $I_{t}=2$ $I_{p} / \lambda \mathrm{kg} \mathrm{m}^{2}$. The O's indicate the beginning of the locus at $\Omega=0$. The $\times$ 's indicate the position of the roots at the point where the system first becomes unstable at $\Omega=2435 \mathrm{rpm}$.

Figure 6 illustrates a case where the criterion (9) is not met, yet the lateral mode still controls stability.

\section{ROTOR AXIAL ASYMMETRY: COUPLING OF THE MODES}

The introduction of asymmetry in either geometric or bearing parameters causes a coupling of the lateral and pivotal modes. The purpose of this

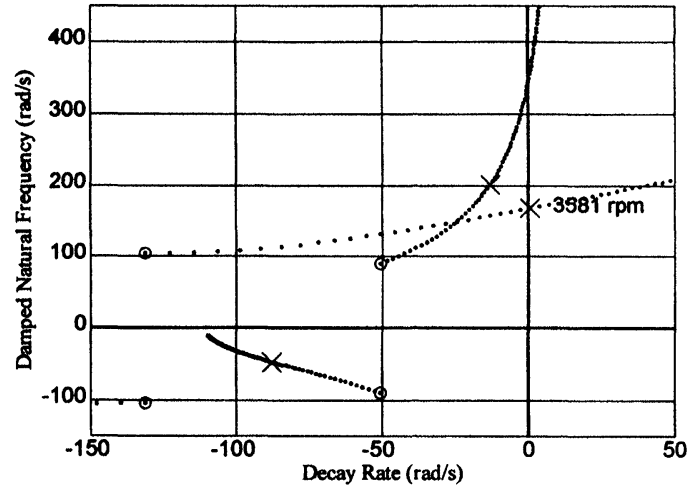

FIGURE 6 Root locus plot of symmetric rotor with system parameters as in Figure 4, except for the parameter $I_{t}=1.1$ $I_{p} / \lambda \mathrm{kg} \mathrm{m}^{2}$. The O's indicate the beginning of the locus at $\Omega=0$. The $\times$ 's indicate the position of the roots at the point where the system first becomes unstable at $\Omega=3581 \mathrm{rpm}$.

investigation is to determine if there is ever an improvement in the rotor stability from asymmetry. The deviations of the rotor system from the mirror symmetric case can be expressed by two coefficients:

\section{Coefficient of geometric asymmetry $a$}

The distances from the center of mass to the bearings, $z_{1}$ and $z_{2}$, can be expressed in terms of 
TABLE II Transformation of nondimensionalization parameters by asymmetry factors

\begin{tabular}{lc}
\hline Lateral natural frequency, $\nu_{t}$ & $\nu_{t}=\sqrt{\kappa / M}$ \\
Natural frequency ratio, $\eta$ & $\eta=L /(2 \rho \sqrt{k}) \sqrt{1+a^{2}-2 a b}$ \\
Lateral damping factor, $\zeta_{\mathrm{t}}$ & $\zeta_{t}=D /(2 \sqrt{K M})$ \\
Angular damping factor, $\zeta_{\theta}$ & $\zeta_{\theta}=\zeta_{t} \eta$ \\
Damping cross coupling factor, $b_{t \theta}$ & $b_{t \theta}=2 \zeta_{t} a_{t \theta}$ \\
Stiffness cross coupling factor, $a_{t \theta}$ & $a_{t \theta}=L /(2 \rho)(a-b)$ \\
\hline
\end{tabular}

the total distance between the bearings, $L$, and an asymmetry factor, $a$, as follows:

$$
z_{1}=(a-1) \frac{L}{2}, \quad z_{2}=(1+a) \frac{L}{2} .
$$

If $a=0$, the rotor system is geometrically symmetric, if $|a|>1$ then the rotor has overhung design (i.e. the rotor center of mass lies outside of the two bearing supports).

\section{Coefficient of stiffness asymmetry $b$}

Similarly, the fluid film direct stiffness' $K_{\mathrm{b} 1}$ and $K_{\mathrm{b} 2}$ can be parametrized in terms of the total stiffness $K=K_{\mathrm{b} 1}+K_{\mathrm{b} 2}$, and an asymmetry parameter, $b$, as follows:

$$
K_{\mathrm{b} 1}=(1+b) \frac{K}{2}, \quad K_{\mathrm{b} 2}=(1-b) \frac{K}{2} .
$$

The same parameter, $b$, can be used to describe the damping asymmetry based on the assumption that the fluid film damping is proportional to stiffness. $D$ represents the total damping $D_{\mathrm{b} 1}+D_{\mathrm{b} 2}$.

$$
D_{\mathrm{b} 1}=(1+b) \frac{D}{2}, \quad D_{\mathrm{b} 2}=(1-b) \frac{D}{2} .
$$

The parameter $b$ can range from 0 to 1 . A symmetric system corresponds to $b=0$. The nondimensional parameters listed in Table I are now transformed in terms of the asymmetry parameters, as follows in Table II:

Taking the relations in Table II into account, the characteristic equation for Eqs. (3) and (4) can be presented in the following nondimensional format:

$$
\begin{gathered}
{\left[s^{2}+2 \zeta_{\mathrm{t}} s+\left(1-2 \mathrm{j} \zeta_{\mathrm{t}} \lambda \omega\right)\right]\left[s^{2}+\left(2 \eta \zeta_{\mathrm{t}}-\mathrm{j} \frac{\omega}{\kappa}\right) s+\eta^{2}\right.} \\
\left.-2 \mathrm{j} \zeta_{\mathrm{t}} \eta \lambda \omega\right]-\frac{a_{\mathrm{t} \theta}^{2}}{\kappa}\left[1+2 \zeta_{\mathrm{t}}(s-\mathrm{j} \lambda \omega)\right]^{2}=0,
\end{gathered}
$$

where $s$ is an eigenvalue. This is a fourth-order equation and consequently has four roots, two of which can have positive real parts. The following expression represents the relation for the instability threshold of the system:

$$
\Omega_{\mathrm{th}}^{(\mathrm{t}, \theta)}=\frac{\nu_{\mathrm{t}}}{\lambda} \sqrt{\frac{1}{2}+\frac{\eta^{2}}{2 \sigma} \pm \sqrt{\frac{1}{4}\left(1-\frac{\eta^{2}}{\sigma}\right)^{2}+\frac{a_{\mathrm{t} \theta}^{2}}{\kappa \sigma}}}
$$

where $\sigma$ is related to the stability criterion given by (9) as follows:

$$
\sigma=1-\frac{I_{\mathrm{p}}}{\lambda I_{\mathrm{t}}} .
$$

If $\sigma>0$, the system has a finite pivotal mode instability threshold. $\sigma \leq 0$ corresponds to infinite stability of the pivotal mode. Note that expression (14) for $a_{\mathrm{t} \theta}=0$ turns into instability thresholds (7) and (8) for the mirror symmetric case. Depending on the parameters, there can be instability thresholds corresponding to either the lateral or pivotal modes. The effects of asymmetry on the instability threshold can now be investigated.

Figure 7 shows a family of curves, each representing a constant value of the fluid film asymmetry parameter, $b$. The stability criterion for the pivotal mode (9) is not met here, consequently the pivotal mode is the stability controlling factor. In this case 

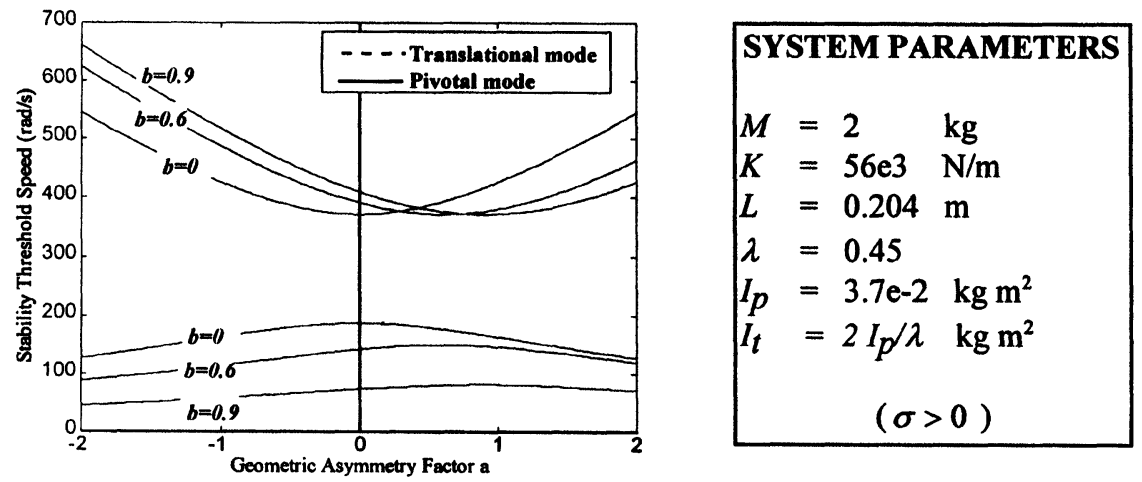

FIGURE 7 Instability threshold versus geometric parameter asymmetry.
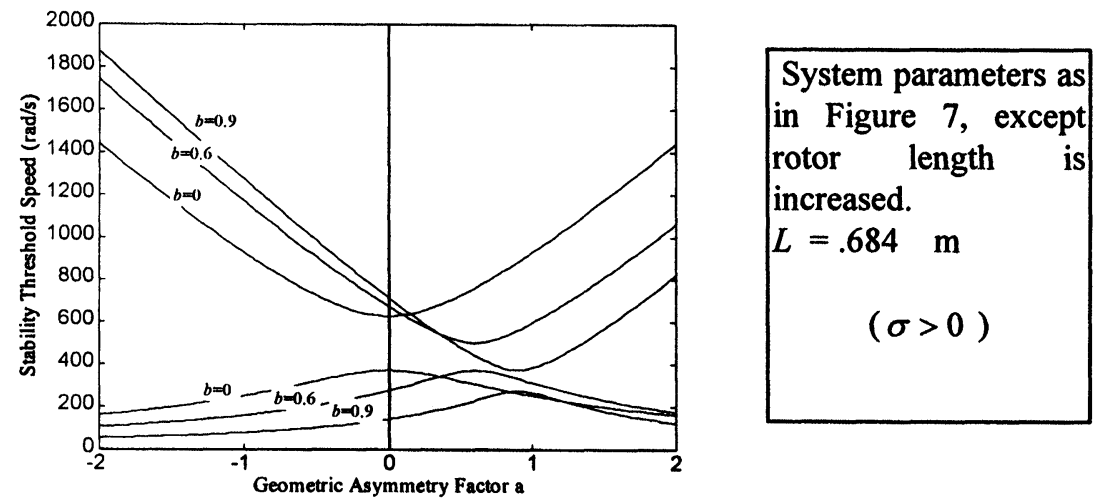

FIGURE 8 Instability threshold versus geometric parameter asymmetry.

it can be seen that asymmetry in either $a$ or $b$ can have only detrimental effects on the instability threshold.

The next case investigated is shown in Fig. 8. All the parameters are identical with Fig. 7, except that rotor length, $L$, is increased. The important insight gained from this case is that in all cases the instability threshold is higher than with a short bearing span. Additionally, note that the peak instability threshold at $b=a=0.6$ is the same as with a symmetric system $b=a=0$. Peak stability is reduced at greater values of fluid film asymmetry, $b=0.9$, yet is still improved over the short rotor case.

Figure 9 shows the case where the stability criterion (9) is satisfied. The rotor length, $L$, is returned to the original smaller value used in Fig. 7. Remarkable about this case is the fact that the peak instability thresholds are equal regardless of $b$ and that the peak value of stability is reached at $a=b$. The translational mode is now entirely responsible for determining stability.

Figure 10 is similar to Fig. 9 with the exception that rotor length, $L$, has been increased. At first glance, the results appear identical because the peak values are the same, but closer inspection will show that the curves take slightly different paths to arrive at the peak values. From this it can be concluded that lengthening the rotor does not have the same benefits when the gyroscopic mode is not the controlling factor for stability. 


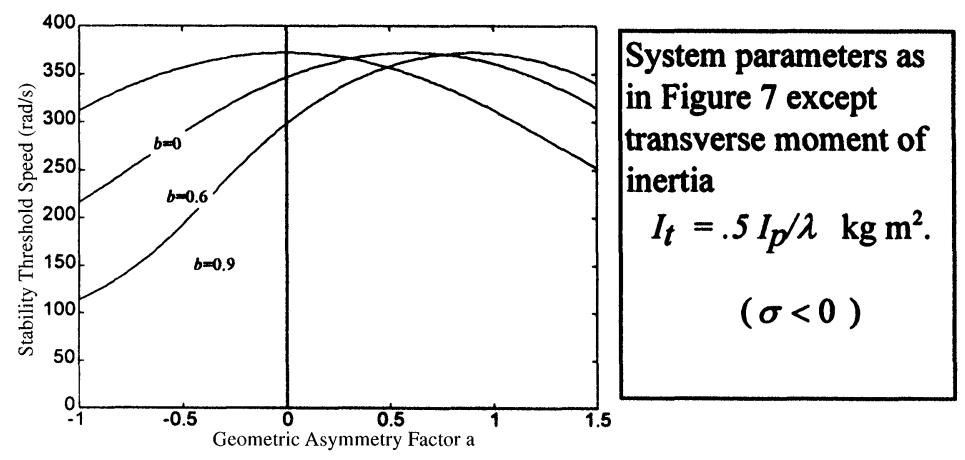

FIGURE 9 Instability threshold versus geometric parameter asymmetry.

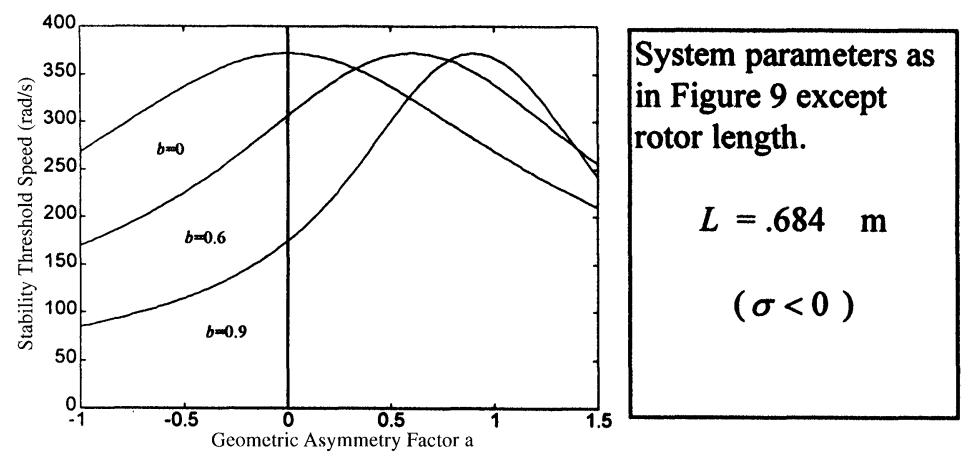

FIGURE 10 Instability threshold versus geometric parameter asymmetry.

\section{CONCLUSIONS}

The rotor system considered is a rigid rotor supported in two fluid film bearings. The latter are assumed to have isotropic stiffness and rotating damping characteristics as by Muszynska (1988). This model, based on the strength of circumferential flow, describes the "cross-coupled stiffness coefficient" as a product of damping, rotative speed, and $\lambda$. This model was identified by extensive modal testing of rotor with fluid interaction by Muszynska (1995). The application of such a model allows a complete parametric stability analysis of the system.

Consideration of stability of the steady state regime in the rotor system with "mirror symmetry" allows for three basic cases:

(i) Highly gyroscopic rotor with high transverse moment of inertia. There are two instability thresholds: one for both translational and pivotal modes. The latter as the lowest controls the rotor stability.

(ii) The rotor with relatively low gyroscopic effect and high transverse moment of inertia: Again there are two instability thresholds, but in this case the translational mode threshold is the lowest, thus controlling overall stability.

(iii) The case of a rotor with relatively low transverse moment of inertia. In this case the pivotal mode is always stable and the rotor stability is determined by the translational mode instability threshold.

The parametric study of the asymmetric rotor system based on the results of the symmetric system stability analysis yields that for the first case the pivotal mode controls the stability for any set of asymmetry parameters. For a given stiffness asymmetry parameter the maximum instability threshold 
is achieved when the rotor center of mass is shifted away from the stiffer bearing on the geometric asymmetry parameter of the same value. The instability threshold maximum corresponds to the symmetric rotor.

This implies that stability may be maximized in this case by distributing the mass of the rotor in such a way as to minimize the ratio of transverse moment of inertia to polar moment of inertia, $\kappa$.

In the case where the translational mode is controlling stability, The instability threshold can only be managed by manipulation of the bearing parameters or total rotor mass.

\section{NOMENCLATURES}

\begin{tabular}{|c|c|}
\hline$a$ & geometric asymmetry parameter \\
\hline$a_{\mathrm{t} \theta}$ & stiffness cross-coupling parameter \\
\hline$b$ & stiffness asymmetry parameter \\
\hline$b_{\mathrm{t} \theta}$ & damping cross-coupling parameter \\
\hline$D_{\mathrm{b} 1}, D_{\mathrm{b} 2}$ & $\begin{array}{l}\text { bearing } 1 \text { and } 2 \text { fluid film damping, } \\
\text { respectively }\end{array}$ \\
\hline$D$ & total lateral fluid film damping \\
\hline$I_{\mathrm{t}}, I_{\mathrm{p}}$ & $\begin{array}{l}\text { transverse and polar moments of } \\
\text { inertia, respectively }\end{array}$ \\
\hline$K_{\mathrm{b} 1}, K_{\mathrm{b} 2}$ & $\begin{array}{l}\text { bearing } 1 \text { and } 2 \text { direct fluid film } \\
\text { stiffness }\end{array}$ \\
\hline$K$ & total lateral fluid film stiffness \\
\hline$z_{1}, z_{2}$ & bearing 1 and 2 location, respectively \\
\hline$L$ & total distance between the bearings \\
\hline$M$ & rotor system mass \\
\hline $\mathrm{O}$ & absolute coordinate system origin \\
\hline$O_{r}$ & rotor center of mass \\
\hline$s$ & eigenvalue \\
\hline
\end{tabular}

$r=x+\mathrm{j} y \quad$ lateral displacement of rotor center of mass in the stationary system of coordinates

$\lambda \quad$ fluid bearing circumferential average velocity ratio

$\chi, \psi \quad$ angle of yaw and pitch, respectively

$\Psi=\chi+\mathrm{j} \psi \quad$ complex angular displacement

$\Omega \quad$ rotative speed

$\Omega_{\mathrm{th}}^{(\mathrm{t}, \theta)} \quad$ instability thresholds for translation

$\mathrm{j}=\sqrt{-1}$ and pivotal modes

\section{References}

Bently, D.E. et al. (1986). Identification of the modal parameters by perturbation testing of a rotor with strong gyroscopic effect, Proc. of International Conference on Rotordynamics, Tokyo, Japan.

Brosens, P.J. and Crandall, S.H. (1982). Whirling of unsymmetrical rotors, Journal of Applied Mechanics, Paper 61-APM-10

Crandall, S.H. and Karnopp, D.C. (1961). Dynamics of Mechanical and Electromechanical Systems, Krieger Publishing Co., Malabar, Fl.

Dimentberg, F.M. (1961). Flexural Vibrations of Rotating Shafts, Butterworths, London.

Ehrich, F.E. (1992). Handbook of Rotordynamics, McGraw-Hill.

Hatch, C.T. and Bently, D.E. (1995). Moment equation representation and stability analysis of a 1-CDOF overhung rotor model with fluid bearing and gyroscopic effects, BRDRC Report No. 8

Muijderman, E.A. (1986). Algebraic formulas for the threshold and mode of instability and the first critical speed of a simple flexibly supported (overhung) rotor-bearing system, Proc. of the International Conference on Rotordynamics, Tokyo, Japan, p. 201.

Muszynska, A. (1988). Improvements in lightly loaded rotor/ bearing and rotor/seal models, Trans. ASME Journal of Vibration and Acoustics, 110(2), 129-136.

Muszynska, A. (1995). Modal testing of rotors with fluid interaction, International Journal of Rotating Machinery, $\mathbf{1}(2), 83-116$.

Vance, J.M. (1988). Rotordynamics of Rotating Machinery, John Wiley \& Sons, New York.

Yamamoto, T. (1954). On the critical speeds of a shaft, Memoirs of the Faculty of Engineering, Nagoya University. 


\section{ait \\ ENERGY MATERIALS}

M A N E Y publishing

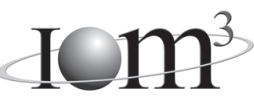

\section{Materials Science \& Engineering for Energy Systems}

Maney Publishing on behalf of the Institute of Materials, Minerals and Mining

The Institute of Materials, Minerals \& Mining

Economic and environmental factors are creating ever greater pressures for the efficient generation, transmission and use of energy. Materials developments are crucial to progress in all these areas: to innovation in design; to extending lifetime and maintenance intervals; and to successful operation in more demanding environments. Drawing together the broad community with interests in these areas, Energy Materials addresses materials needs in future energy generation, transmission, utilisation, conservation and storage. The journal covers thermal generation and gas turbines; renewable power (wind, wave, tidal, hydro, solar and geothermal); fuel cells (low and high temperature); materials issues relevant to biomass and biotechnology; nuclear power generation (fission and fusion); hydrogen generation and storage in the context of the 'hydrogen economy'; and the transmission and storage of the energy produced.

As well as publishing high-quality peer-reviewed research, Energy Materials promotes discussion of issues common to all sectors, through commissioned reviews and commentaries. The journal includes coverage of energy economics and policy, and broader social issues, since the political and legislative context influence research and investment decisions.

\section{CALL FOR PAPERS}

Contributions to the journal should be submitted online at http://ema.edmgr.com

To view the Notes for Contributors please visit: www.maney.co.uk/journals/notes/ema

Upon publication in 2006, this journal will be available via the Ingenta Connect journals service. To view free sample content online visit: www.ingentaconnect.com/content/maney

For further information please contact:

Maney Publishing UK

Tel: +44 (0)113 2497481 Fax: +44 (0)1132486983 Email: subscriptions@maney.co.uk

or

Maney Publishing North America

Tel (toll free): 8662975154 Fax: 6173546875 Email: maney@maneyusa.com

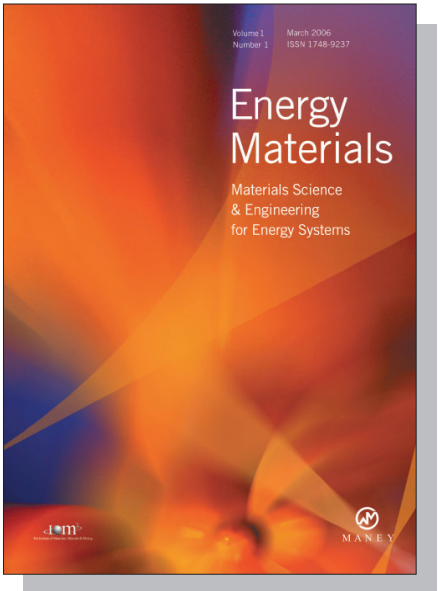

EDITORS

Dr Fujio Abe

NIMS, Japan

Dr John Hald, IPL-MPT, Technical University of Denmark, Denmark

Dr R Viswanathan, EPRI, USA

\section{SUBSCRIPTION INFORMATION}

Volume 1 (2006), 4 issues per year

Print ISSN: 1748-9237 Online ISSN: 1748-9245

Individual rate: $£ 76.00 / U S \$ 141.00$

Institutional rate: $£ 235.00 /$ US $\$ 435.00$

Online-only institutional rate: $£ 199.00 / U S \$ 367.00$

For special $\mathrm{IOM}^{3}$ member rates please email

subscriptions@maney.co.uk

\section{For further information or to subscribe online please visit www.maney.co.uk}



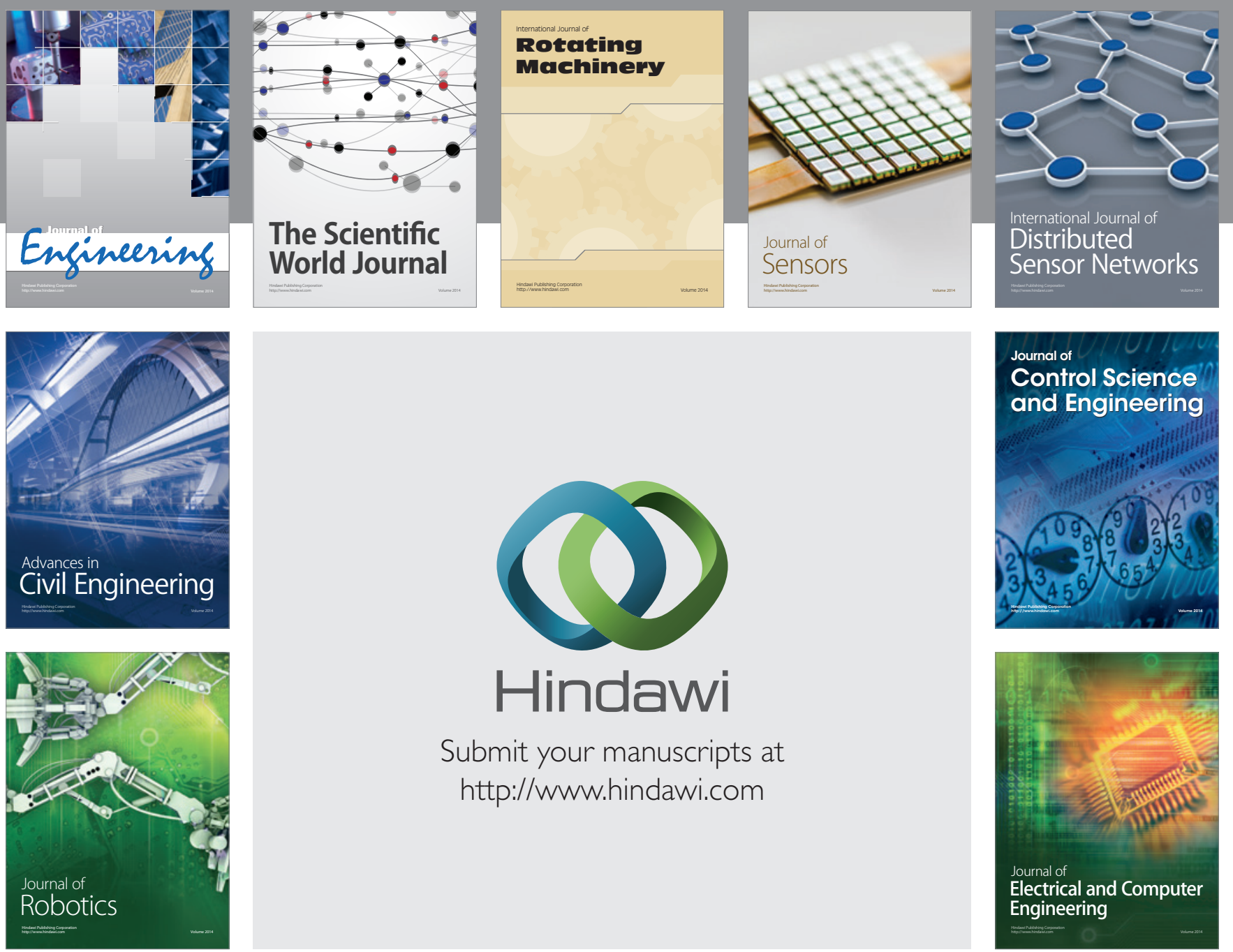

Submit your manuscripts at

http://www.hindawi.com
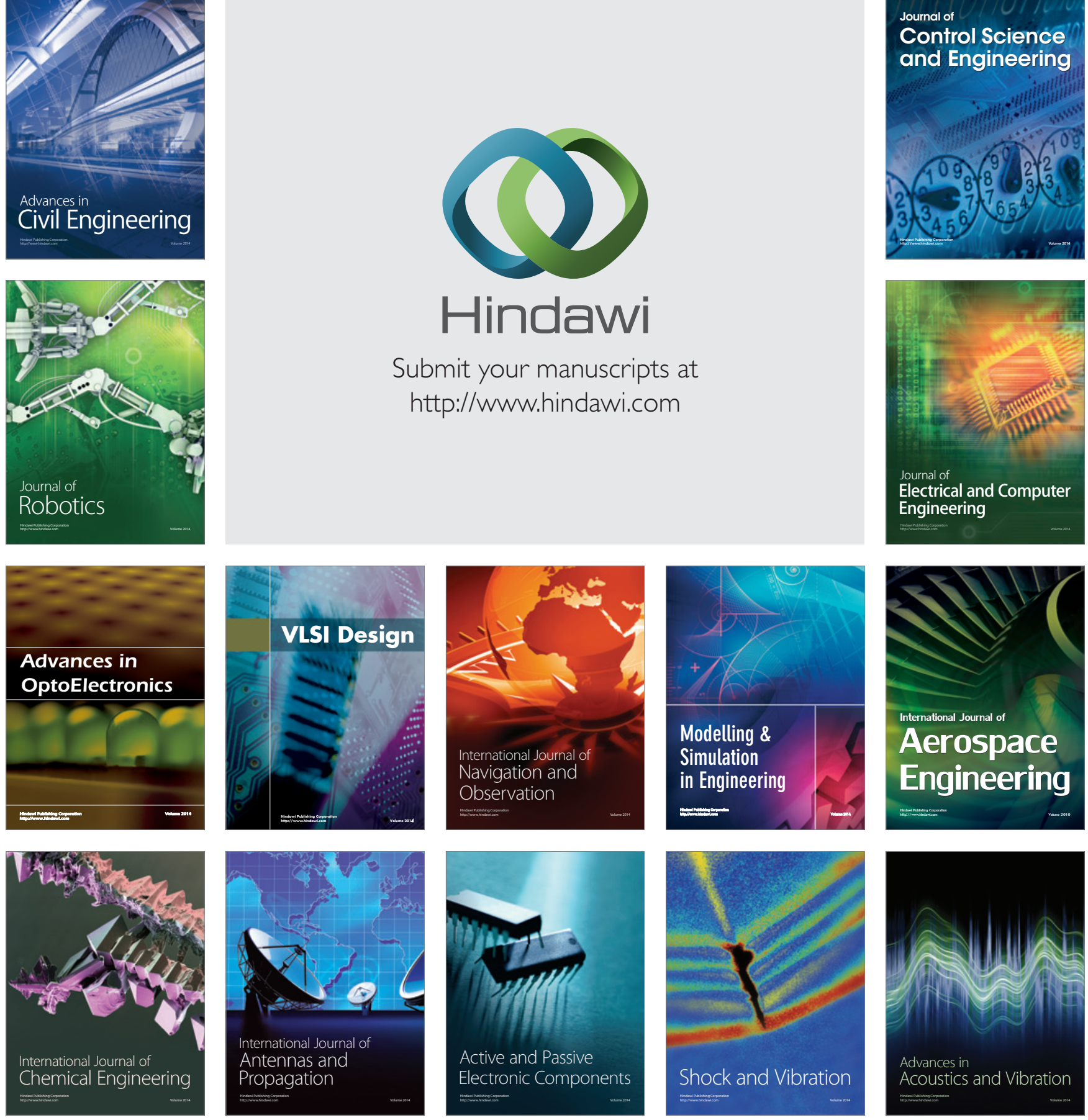